\title{
EDITORIAIS
}

\section{Os usos inadequados e os efeitos adversos de medicamentos na prática clínica}

\author{
The inappropriate usage and adverse effects of drugs in clinical practice
}

\author{
Anthony Wong*
}

\begin{abstract}
As questões abordados neste número por Carvalho et al. levantam uma parte dos problemas e decisões que constantemente afligem não apenas os médicos que trabalham em terapia intensiva, mas também em serviços de emergência e no consultório ${ }^{1}$. $\mathrm{O}$ uso de medicamentos contrários e não-condizentes com as recomendações dos órgãos regulatórios, das farmacopéias e das sociedades
\end{abstract} médicas em número tão elevado, apontado neste trabalho, reflete apenas "a ponta do iceberg" desta situação, e que já se tornou uma prática corriqueira e até banalizada. O que é surpreendente é a lista de medicamentos apresentada por estes autores como "não aprovados", que são dois apenas, visto que a dipirona é aprovada no Brasil. Os demais medicamentos não estavam corretos apenas na posologia.

Isso difere substancialmente do que se vê em outros centros nas quais um grande número de medicamentos são usados inadequadamente, dentro e fora das UTIs pediátricas, tais como o uso de antibióticos (e.g. fluoroquinolonas), certos antiinflamatórios, drogas vasoativas e cardioativas e antidepressivos, que não estão autorizados para uso em crianças. Pode esta prática ser justificada pelas tentativas, às vezes desesperadas, de salvar uma vida? Existiriam poucos medicamentos disponíveis e eficazes para pacientes pediátricos? Mesmo nos Estados Unidos, aproximadamente $50 \%$ a $75 \%$ dos remédios usados na clínica pediátrica não foram avaliados adequadamente ${ }^{2}$. A experiência mostra que muitos remédios podem ser usados com segurança em

* Doutor em Medicina pela Faculdade de Medicina da Universidade de São Paulo. Médico chefe do CEATOX - Centro de Assistência Toxicológica do Instituto da Criança do Hosp. das Clínicas (FMUSP) e Centro de Referência em Farmacovigilância da Organização Mundial da Saúde. Diretor do IBET - Instituto Brasileiro de Estudos em Toxicologia e Farmacologia. crianças, se por curto espaço de tempo. Porém, nestes casos, seu uso empírico e sem nenhum critério científico deixa o pediatra a mercê de dúvidas e sujeito a críticas.

Levantamentos bibliográficos mostram que os incentivos e legislações que promovem ensaios clínicos em crianças auxiliaram o melhor conhecimento da farmacocinética e forneceram importantes informações da dose e da segurança de medicamentos usados em pacientes pediátricos ${ }^{2}$. Em 1997, e novamente em 2002, o Congresso americano estendeu a patente de um medicamento se este tivesse ensaios clínicos pediátricos. Em 1998, o FDA exigia ensaios pediátricos em qualquer medicamento que tivesse potencialmente uso pediátrico. A Academia Americana de Pediatria estabeleceu normas e critérios éticos para ensaios envolvendo crianças ${ }^{3}$. Tais iniciativas não foram ainda adotadas no nosso país.

Por outro lado, é necessário enfatizar que o uso indiscriminado de remédios e das associações de fármacos aumenta a morbi-mortalidade devido aos eventos adversos e à toxicidade destes, com um custo elevadíssimo, superior a 3 bilhões de dólares anualmente nos EUA ${ }^{4}$. Segundo um estudo da Harvard, as reações adversas naquele país são muito mais comuns entre pacientes hospitalizados, podendo superar inclusive os acidentes automobilísticos e a violência ${ }^{5}$. A incidência de efeitos adversos por medicamentos na idade pediátrica é de $4,5 \%$ a $9,8 \%$. Pode ser até maior do que em adultos, e a maior incidência de eventos adversos e erros de medicação ocorria principalmente em recém-nascidos, especialmente nas terapias intensivas neonatais ${ }^{6}$. No Brasil, não existe levantamento semelhante, mas pode-se suspeitar que o hábito de automedicação, o freqüente uso de associações e o grande número de medicamentos (alopáticos, homeopáticos e fitoterápicos) disponíveis possam causar prejuízos econômicos, sociais e à saúde ainda mais significativos. 
A incidência de eventos adversos aumenta exponencialmente com o número de medicamentos administrados durante um período, mas não necessariamente ao mesmo tempo ${ }^{7}$. A tabela abaixo demonstra que o uso de $20 \mathrm{ou}$ mais remédios aumenta o risco de reação adversa em $45 \%$, ocorrência esta relativamente freqüente em UTIs, e mesmo nas enfermarias onde há pacientes com internação prolongada.

\begin{tabular}{cc}
\hline Número de drogas & \% de reações adversas \\
\hline $0-5$ & 4,2 \\
$6-10$ & 7,4 \\
$11-15$ & 24,2 \\
$16-20$ & 40 \\
$>21$ & 45 \\
\hline
\end{tabular}

Smith JW et al., $1966^{7}$.

Trabalho publicado por residentes do Hospital Johns Hopkins indicou que 7,5\% das doses dos medicamentos administrados resultaram em efeitos nocivos clinicamente relevantes ${ }^{8}$.

É igualmente preocupante a constatação do desconhecimento sobre reações adversas e riscos da interação de drogas entre os nossos médicos e doutorandos. Pesquisa que realizamos entre formandos de uma das melhores faculdades de medicina do país indicou que mais de $90 \%$ destes não conseguiam identificar graves interações de drogas usadas corriqueiramente. Um questionário realizado entre médicos de um grande hospital público paulistano demonstrou que mais da metade deles não conseguia definir nem identificar efeito adverso e interação medicamentosa.

Estes fatos indicam a necessidade premente de intensificar a preparação dos nossos médicos na arte de prescrever e curar, recebendo melhores informações e dispondo de medicamentos seguros e eficazes, que foram submetidos a ensaios clínicos de boa qualidade, em especial para os pacientes pediátricos.

O Dr. Peter Budetti, da Universidade de Oklahoma, afirma em editorial que é essencial conduzir testes de fármacos para uso em pacientes pediátricos, que é necessário instruir os médicos e assegurar que as crianças serão beneficiadas pelos medicamentos que recebem, e que os tempos quando se prescrevia para crianças através de tentativa e erro não deveriam existir mais ${ }^{9}$.

\section{Referências bibliográficas}

1. Carvalho PR, Carvalho CG, Alievi PT, Martinbiancho J, Trotta EA. Identificação de medicamentos "não-apropriados para crianças" em prescrições de Unidade de Tratamento Intensivo Pediátrica. J Pediatr (Rio J) 2003;79:397-402.

2. Roberts R, Rodriguez W, Murphy D, Crescenzi T. Pediatric drug labeling: improving the safety and efficacy of pediatric therapies. JAMA 2003;290(7):905-11.

3. Committee on Drugs, American Academy of Pediatrics. Guidelines for the ethical conduct of studies to evaluate drugs in the pediatric populations. Pediatrics 1995;95:286-94.

4. Oliveira GG. As reações adversas medicamentosas (RAM): o risco terapêutico. A Folha Médica 1994;109(1):47-50.

5. Brennan TA, Leape LL, Laird NM, Lawthers AG, Localio AR, Barnes BA, et al. Incidence of adverse events and negligence in hospitalized patients: results of the Harvard Medical Practice Study I. N Engl Med J 1991;324:370-6.

6. Kauchal R, Bates DW, Landrigan C, McKenna KJ, Clapp MD, Federico F, et al. Medication errors and adverse drug events in pediatric inpatients. JAMA 2001;285:2114-20.

7. Smith JW, Seidl LG, Cluff LE. Studies on the epidemiology of adverse drug reactions V. Clinical factors influencing susceptibility. Ann Intern Med 1966;65:629-40.

8. Seidl LG, Thornton G, Smith JW, Cluff LE. Studies on the epidemiology of adverse drug reactions. III. Reactions in patients on a general medical service. Bull Johns Hopkins Hosp 1966;119:299-315.

9. Budetti PP. Ensuring safe and effective medications for children. JAMA 2003;290(7):950-2. 